\title{
Brucellosis Relapse: A Retrospective Study of Risk Factors among Saudi Patients
}

\author{
Elsayed Saad Abd elbaser ${ }^{1}$ and Heba A. Mohammed ${ }^{2}$ \\ Departments of Tropical Medicine ${ }^{l}$ and Microbiology and Immunology ${ }^{2}$, \\ Faculty of Medicine, Zagazig University, Zagazig, Egypt
}

\section{Corresponding Author Elsayed Saad Abd- elbaser}

Mobile: 00201094986320

E mail: dr.sayedsaad79@gmai l.com

Key words: Brucellosis; Relapse; Risk factors
Background and study aim: Brucellosis is one of the most common zoonotic infections worldwide. Brucella organisms are able to survive and even multiply within mononuclear cells explaining the tendency of the disease to have a prolonged clinical course and relapse. The incidence of relapse in Brucellosis remains high; ranging from $5-30 \%$ of patients. The aim of this study is to evaluate possible risk factors of Brucellosis relapse.

Patients and Methods: A retrospective study of 127 patients was conducted to evaluate risk factors of Brucellosis relapse. The diagnosis of relapse was based on recurrence of clinical picture within 3-6 months from treatment, increase agglutinating antibody titers and PCR.
Different demographic, clinical, laboratory and serological parameters were studied based on the data gathered from the patients`electronic files.

Results: Out of 127 patients, 21 (16.5\%) patients were diagnosed as relapsers. No significant differences were found between relapsed and non-relapsed patients regarding age, sex, residence, clinical features, complications, lymphocytic count and serological titers. As regard treatment, regimens included Streptomycin and Doxycycline had a significant decrease in relapse occurrence.

Conclusion: Anti-microbial regimen included Streptomycin and Doxycycline is the only preventive factor for Brucellosis relapse.

\section{INTRODUCTION}

Brucellosis is one of the most common zoonotic infections worldwide with more than 500.000 new cases reported to World Health Organization (WHO) every year [1]. It is still endemic in certain countries of Middle East, among these countries is Saudi Arabia, since it hosts a myriad of travellers and imports a high number of livestock [2]. Occupational exposure is the most frequent route of infection, thorough inhalation of infectious aerosols, so it is common among shepherds, farmers and laboratories' staff. Non-occupational sources of infection include the consumption of un-pasteurized milk, goat cheese and raw meat [3].

Definite diagnosis of Brucellosis requires isolation of the organism from the blood or other body fluids, but serological methods are rapid, easy to perform and may be the only tests available in certain settings [4]. Identification of specific antibodies can be done by standard agglutination test (SAT) or 2 mercaptoethanol (2-ME). SAT is the most common serological test used in endemic countries with a titer $\geq 1 / 160$ is considered significant in non-endemic areas and $\geq 1 / 320$ in endemic areas [5].

Brucella organisms are able to survive and even multiply within mononuclear cells explaining the tendency of the disease to have a prolonged clinical course and relapse [6]. Even with the correct treatment, the incidence of relapse in Brucellosis remains high, ranging from $5-30 \%$ of patients [7]. Diagnosis of relapse is difficult, since blood culture has low sensitivity (50$65 \%$ ) and serological tests may remain positive in patients with no evidence of clinical relapse. Polymerase chain reaction (PCR) is a more sensitive technique for early detection of relapse [8]. Risk factors of relapse are not yet clearly understood, but it may be attributed to; antibiotic regimen, incomplete therapy, depressed cell 
mediated immunity (CMI), long duration between appearance of symptoms and initiation of treatment, complications and Brucella species $[\mathbf{9 , 1 0}]$. The aim of this study is to evaluate these factors and other demographic, clinical and serological factors, searching for possible factors of relapse of Brucellosis.

\section{PATIENTS AND METHODS}

This retrospective study was done based on the files of 127 patients, who were diagnosed with Brucellosis, at Al Amen Hospital, Taif, Saudi Arabia, during the period from October 2013 to October 2016.

\section{Inclusion criteria:}

1. Active Brucellosis

2. Age above 18 years

\section{Exclusion criteria:}

1- Incomplete treatment

2- Incomplete follow up

3- Failure of treatment

The diagnosis of Brucellosis was based on the presence of compatible clinical features; fever, myalgia, sweating and backache together with the demonstration of significant antibody titers; SAT $>1: 320$ and 2-ME $>1: 160$.

These patients were treated according to the following regimens :

- Regimen (1) included combination of streptomycin 1gm once daily and doxycycline 100 $\mathrm{mg}$ twice/day for 21 days, then doxycycline $100 \mathrm{mg}$ twice/day and rifampicin $600 \mathrm{mg}$ daily for another 21 days.

- Regimen (2) included doxycycline 100mg twice/ day and streptomycin $1 \mathrm{gm} /$ day for 6 weeks.

- Regimen (3) included doxycycline 100mg twice/ day and ofloxacin $200 \mathrm{mg}$ daily for 4 weeks.

- Regimen (4) included rifampicin $600 \mathrm{mg} /$ day and co-trimoxazole (80 mg TMP/400 mg SMZ) for 4 weeks.

- Regimen (5) included other antimicrobials.

The diagnosis of relapse was based on recurrence of clinical picture within 3-6 months from treatment; increase agglutinating antibody titers which was decreased previously and confirmed by PCR.
For each patient the following data were collected from electronic files: age, sex, residency (rural, urban), occupational exposure, clinical features (fever, body aches, myalgia, backache), laboratory results (CBC, Wright, 2ME) and antimicrobial regimens.

\section{Statistical analysis:}

All the data were registered in special forms designed for this study. The SPSS version 16 was used for statistical analysis. Chi-square test was used for comparing data of both groups. P value of $<0.05$ indicates significant results.

\section{RESULTS}

Out of 127 studied patients, 104 (82\%) were males and $23(18 \%)$ were females. Twenty one (16.5\%) patients were diagnosed as relapsers. The mean age for relapsed patients was $41.09 \pm 17.43$ and for non-relapsed patients was $39.2 \pm 19.6$. About $32(25 \%)$ patients were living in urban areas and $95(75 \%)$ were in rural areas. Of all patients, 50 (39.3\%) patients had contact to animals.

Regarding clinical symptoms; out of 77 (60.6\%) patients who had fever, 14 (18\%) patients showed a relapse. Moreover, 62 (49\%) patients had sweating, of them $9(14.5 \%)$ patients had a relapse. Considering myalgia and backaches, there were 108 and 49 patients respectively complaining, of them $19(17.6 \%)$ and $9(18.3 \%)$ patients respectively had a relapse.

There was non-significant difference between both groups regarding complications, 33 (26\%) patients had complications, of them 5 (15\%) patients had a relapse. Furthermore, Wright test was <1:640 in 65 patients, of them 10 (15.3\%) patients showed a relapse, while those with Wright test $>1: 640$ were 62 patients, of them 11 (18\%) patients had a relapse.

Out of 127 studied patients, 80 (63\%) patients were treated with streptomycin and doxycycline, of them $5(6.2 \%)$ patients relapsed. On the other hand, $40(31.5 \%)$ patients were treated with doxycycline and ofloxacin or rifampicin and cotrimoxazole, of them 13 (32.5\%) patients relapsed. Regimen No. 1 and 2 showed statistically significant differences between relapsed and nonrelapsed patients while other regimens showed no differences (Figures 1 and 2). 


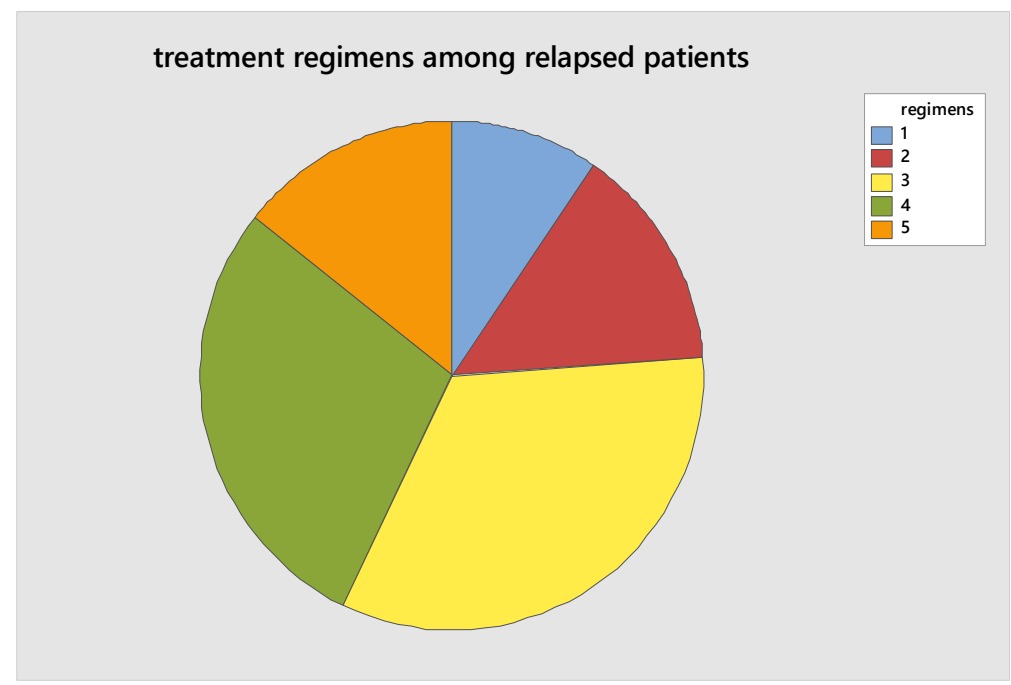

Figure (1): Treatment regimens among relapsed patients. 1; doxycycline, streptomycin and rifampicin 2; doxycycline, rifampicin 3; doxycycline, ofloxacin 4; rifampicin, co-trimoxazole 5; others

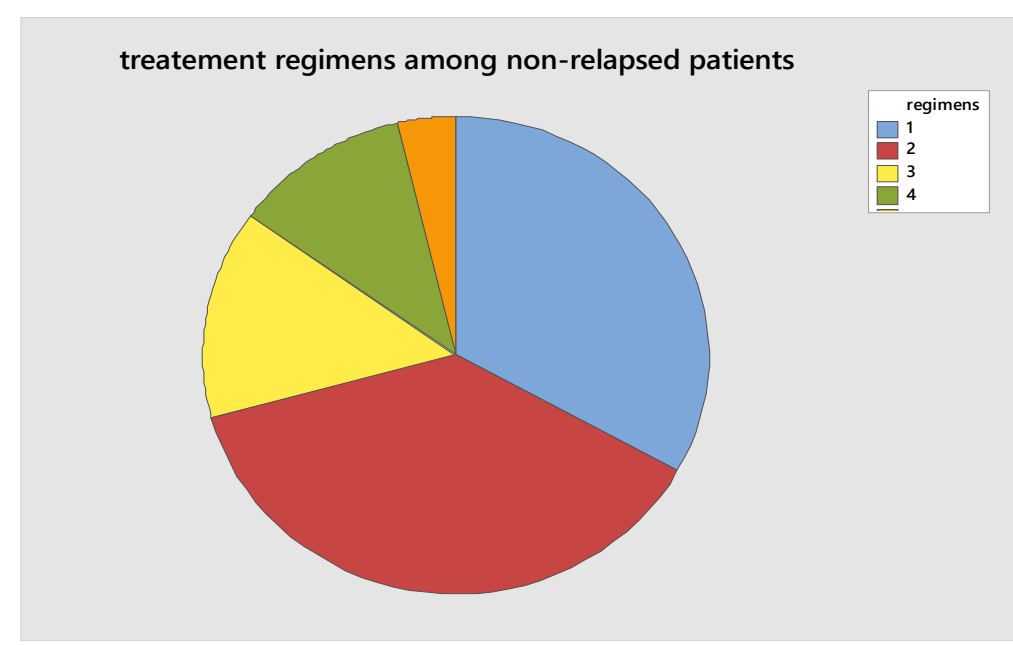

Figure (2): Treatment regimens among non-relapsed patients.1; doxycycline, streptomycin and rifampicin 2 ; doxycycline, rifampicin 3; doxycycline, ofloxacin 4; rifampicin, co-trimoxazole 5; others 
Table (1): Demographic, clinical, serological data and treatment regimens among both populations

\begin{tabular}{|c|c|c|c|}
\hline Parameter & $\begin{array}{c}\text { Relapse } \\
(\mathrm{N}: 21) \\
(\mathbf{1 6 . 5 \%}) \\
\end{array}$ & $\begin{array}{c}\text { Non- relapse } \\
(\mathbf{N}: \mathbf{1 0 6}) \\
(\mathbf{8 3 . 5 \%}) \\
\end{array}$ & $\mathbf{P}$ \\
\hline Age & $41.09 \pm 17.43$ & $39.2 \pm 19.6$ & 0.660 \\
\hline $\begin{array}{l}\text { Sex: } \\
\text { Male (N. 104) } \\
\text { Female (N. 23) }\end{array}$ & $\begin{array}{l}19(18 \%) \\
2(8.7 \%)\end{array}$ & $\begin{array}{c}85(82 \%) \\
21(91.3 \%)\end{array}$ & 0.361 \\
\hline $\begin{array}{l}\text { Residency: } \\
\text { Urban (N. 32) } \\
\text { Rural (N. 95) }\end{array}$ & $\begin{array}{l}5(15.6 \%) \\
16(17 \%)\end{array}$ & $\begin{array}{c}27(84.4 \%) \\
79(83 \%)\end{array}$ & 1.00 \\
\hline Occupational (N. 50) & $8(16 \%)$ & $42(84 \%)$ & 1.00 \\
\hline Fever (N. 77) & $14(18 \%)$ & $63(82 \%)$ & 0.629 \\
\hline Sweating (N. 62) & $9(14.5 \%)$ & $53(85.5 \%)$ & 0.636 \\
\hline Myalgia (N. 108) & $19(17.6 \%)$ & $89(82.4 \%)$ & 0.738 \\
\hline Backaches (N. 49) & $9(18.3 \%)$ & $40(81.7 \%)$ & 0.807 \\
\hline Arthralgia (N. 98) & $18(18.3 \%)$ & $80(81.7 \%)$ & 0.401 \\
\hline Complications (N. 33) & $5(15 \%)$ & $28(85 \%)$ & 1.00 \\
\hline $\begin{array}{r}\text { Wright test } \\
\quad<1: 640 \text { (N. 65) } \\
>1: 640 \text { (N. 62) } \\
\end{array}$ & $\begin{array}{c}10(15.3 \%) \\
11(18 \%)\end{array}$ & $\begin{array}{c}55(84.7 \%) \\
51(82 \%)\end{array}$ & 0.813 \\
\hline Lymphocytosis (N. 27) & $5(18.5 \%)$ & $22(81.5 \%)$ & 0.773 \\
\hline $\begin{array}{l}\text { Brucella species: } \\
\text { B. Melitensis (N. 103) } \\
\text { B. abortus (N. 24) } \\
\end{array}$ & $\begin{array}{c}18(17.5 \%) \\
3(12.5 \%) \\
\end{array}$ & $\begin{array}{l}85(82.5 \%) \\
21(87.5 \%) \\
\end{array}$ & 0.763 \\
\hline $\begin{array}{c}\text { Treatment regimen: } \\
\text { No. } 1 \text { (N. } 37) \\
\text { No. } 2 \text { (N. } 43) \\
\text { No. } 3 \text { (N. } 22) \\
\text { No. } 4 \text { (N. } 18) \\
\text { No. } 5 \text { (N. } 7)\end{array}$ & $\begin{array}{c}2(5.4 \%) \\
3(7 \%) \\
7(32 \%) \\
6(33.3 \%) \\
3(43 \%)\end{array}$ & $\begin{array}{c}35(94.6 \%) \\
40(93 \%) \\
15(68 \%) \\
12(66.7 \%) \\
4(57 \%) \\
\end{array}$ & $\begin{array}{r}0.035^{*} \\
0.044^{*} \\
0.054 \\
0.079 \\
0.088\end{array}$ \\
\hline
\end{tabular}

*Significant

\section{DISCUSSION}

The immune responses against Brucellosis include cell mediated immunity mainly and to less extend humoral immunity. On the other hand, it has different mechanisms to evade these immune responses and hence, chronic infection and relapse occur [10]. Chronic infection and relapse are associated with an impaired Th1 response, NK cells cytotoxicity and IFN $\gamma$ production [11]. Relapse of Brucellosis is a very common problem after treatment with single anti-microbial agent and also may be related to incomplete treatment. Other risk factors that were assumed to be responsible for relapse in different studies are; depressed cell mediated immunity (CMI), complications, Brucella species and long duration between appearance of symptoms and initiation of treatment [12-15]. We aimed in this study to evaluate these risk factors in addition to other demographic, serologic and laboratory factors, searching for possible factors responsible for relapse of Brucellosis.

An important issue in studying the risk factors of Brucellosis relapse is the differentiation between relapse and reinfection. Relapse is reappearance of the compatible clinical features within 6 months after conclusion of treatment together with rising serological titers. In endemic areas, like Saudi Arabia, where the re-exposure risk is persistent, relapse is difficult to be differentiated from reinfection. In this study we used the PCR to confirm that the clinical features and rising antibody titer were due to relapse rather than reinfection. Morata et al. [8] reported that PCR is a sensitive method for early detection of relapse.

In this study, the only significant risk factor that might be responsible for relapse was the regimen of treatment. We evaluated 127 patients who were treated by 5 different regimens commonly used 
to treat Brucellosis in Saudi Arabia's community. The two regimens that were significantly found to prevent the relapse are those that included both streptomycin and doxycycline antibiotics (regimens 1 and 2). The duration of both regimens was 6 weeks. On the other hand, the other nonpreventive two regimens (regimens 3 and 4) did not include streptomycin but doxycycline and ofloxacin or rifampicin and co-trimoxazole. The duration was 4 weeks only. WHO has been approved the combination of doxycycline $100 \mathrm{mg}$ twice/day for 6weeks and streptomycin $(1 \mathrm{~g} /$ day intramuscularly) for 2-3 weeks, as the treatment regimen of choice in eradicating Brucella and prevent the relapse [1]. Consistent with these results, Roushan et al. [12] also reported that treatment regimen is the only preventive factor. In their retrospective study the regimens that have been found to prevent relapse included streptomycin or gentamicin and doxycycline.

As regarding other risk factors that were postulated to be responsible for relapse including; lymphocytic count, depressed cell mediated immunity (CMI), complications, Brucella species and long duration between appearance of symptoms and initiation of treatment, there were non-significant differences between both groups regarding these factors. Unfortunately, the duration of symptoms was not reported in some files of our patients, so we couldn't study this factor. Alavi et al. [9] reported that patients with relapsed Brucellosis had lymphopenia, longer duration of symptoms before initiation of treatment and increased titers of antibodies. Solera et al. [13] found that fever, positive blood culture and symptom duration of less than 10 days were associated with relapse, but age, sex, ESR, alanine transaminase (ALT), leukocyte, hemoglobin, platelet, serum albumin, and alkaline phosphatase (ALP) levels were found not to be associated with relapse. Ariza et al. [14] showed that inappropriate antibiotic treatment, prolonged blood culture positivity, pretreatment illness duration shorter than 10 days, and platelet count less than $150,000 / \mathrm{ml}$ were predictive factors of relapse. Aktug demir and Ural [15] studied the relation between cytokines and relapse, they found that relapsed patients has higher interleukin 8 . They also found no significant difference between relapsed and non- relapsed patients regarding lymphocytic count. In Morata et al. [8] 70\% of the patients had duration of symptoms less than 2 months versus $16.6 \%$ had more than 2 months.
From the above reported results, the duration of symptoms before treatment appears to be controversy. While Alavi et al., reported that longer duration of symptoms was associated with relapse, Ariza et al., and Solera et al. $[\mathbf{1 3 , 1 4}]$ reported that symptom duration of less than 10 days was associated with relapse. Unfortunately, we couldn't study this factor. Also, correlation of cytokines to the incidence of relapse was suggested by Aktug demir and Ural. We did not study the role of cytokines, since this study was retrospective. So, we recommend further prospective studies to be carried out and include other risk factors in order to predict relapse and hence prevention by rearranging treatment and monitoring of the patients.

\section{CONCLUSION}

Anti-microbial regimen is the only preventive factor for Brucellosis relapse. This study recommends using combination of streptomycin and doxycycline for treatment of Brucellosis, since it has lower rate of relapse. On the other hand, none of the other studied factors were founded to prevent the relapse.

\section{Funding: None \\ Conflicts of interest: None.}

\section{Ethical approval:Approved}

\section{REFERENCES}

1. Corbel MJ. Brucellosis in humans and animals. 2006. World Health Organization (WHO), ISBN 9241547138 .

2. Abdulaziz D, Aloufi A, Ziad A. Memish AB, Abdullah M. Assiri AB, et al.. McNabb B; Trends of reported human cases of Brucellosis, Kingdom of Saudi Arabia, 2004-2012. Journal of Epidemiology and Global Health, 2016, 6, 11-18

3. AL-EISSA Y.A. Brucellosis in Saudi Arabia: Past, present and future. Ann. Saudi Med., Sept. 2000.

4. Franco MP, Mulder M, Glman RH, Smitset HL. Human Brucellosis. Lancet infect. Dis 2007; 7:775-86.

5. Arag GF. Update on laboratory diagnosis of Brucellosis. Int J Antimicrob Agents 2010, 36 (supplI) S12-17

6. Ocon P, Reguera J M, Morata P, Juarez C, Alonso A, Colmenero J D. Phagocyte cell function in active Brucellosis. Infect. Immun. 1994, 62:910-914 
7. Solera, J. Update on Brucellosis: therapeutic challenges. International Journal of Antimicrobial Agents, 2010, 36, S18-S20

8. Morata P, Queipo-otuno M, Reguera J, Gaciaordonez M, Pichardo C, Colmenero J. Post treatment Follow-Up of Brucellosis by PCR Assay. Journal of Clinical Microbiology. 1999: 4163-4166

9. Alavi S, Alavi S, Alavi L. Relapsed human Brucellosis and related risk factors. Pak J Med Sci. 2009, 25(1): 46-50

10. Skendros P, Pappas G, Boura P. Cell-mediated immunity in human Brucellosis. Microbes Infect 2011; 13: 134-42.

11. Alturi VL, Xavier MN, de Jong MF, den Hartigh $\mathrm{AB}$, Tsolis RM. inter-actions of the human pathogenic Brucella species with their hosts. Annu Rev Microbiol 2011; 65:523-41.
12. Roushan M, Moulana Z, Afshar ZM, Ebrahimpour S. Risk Factors for Relapse of Human Brucellosis. Global Journal of Health Science; 2016; 8, (7)

13. Solera J, Martinez-Alfaro E, Espinosa A, Castillejelos ML, Geijo P, Rodriguez-Zapata M. Multivariate model for predicting relapse in human brucellosis. Journal of Infection 1998; 36: 85-92.

14. Ariza J, Corredoira J, Pallares R, Viladrich PF, Rufi G, Pujol M, et al. Characteristics of and riskfactors for relapse of brucellosis in humans. Clin Infect Dis. 1995; 20: 1241-9

15. Aktug demir N, Ural O.; Serum interleukin-8 levels may predict relapse in brucellosis. Turk $J$ Med Sci 2012; 42 (5): 796-801 